\title{
Editorial
}

Nephrology

\section{Total Kidney Volume and Autosomal Dominant Polycystic Kidney Disease: A Long-Standing Relationship}

\author{
Maria V. Irazabal Vicente E. Torres \\ Department of Internal Medicine, Division of Nephrology and Hypertension, Rochester, MN, USA
}

Autosomal dominant polycystic kidney disease (ADPKD) is the most common monogenic kidney disease and the fourth leading cause of renal failure among adults, affecting approximately 12.5 million people worldwide. Cyst development starts in utero and continues through the patient's lifetime. The median age of endstage renal disease is 54-years for PKD1 and 74-years for $P K D 2$, but the rate of disease progression varies widely among individuals [1]. This large phenotypic variability and the maintenance of normal glomerular filtration rate (GFR) until late stages of the disease represent a major challenge for nephrologists following these patients. At an early stage, it is difficult to predict disease progression or evaluate a new therapy based solely on renal function markers. On the other hand, if new therapies are implemented when GFR starts to decline and irreversible damage has occurred, they are less likely to be effective. With the recent Food and Drug Administration (FDA) approval of tolvaptan (a vasopressin V2 receptor antagonist), and other therapies undergoing clinical testing, it is imperative to identify patients' disease severity and their risk of progression, to select the best candidates for therapy and assess a treatment response.

Advances in imaging techniques and results from the Consortium for Radiologic Imaging Studies of Polycystic Kidney Disease (CRISP) observational cohort study shed

\section{KARGER}

(c) 2018 S. Karger AG, Basel

E-Mail karger@karger.com

www.karger.com/ajn light into how cysts develop and grow and the progression of the disease. They found that kidney and cyst volumes increase in most patients at an average rate of $\sim 5 \%$ per year, and that larger kidneys are associated with a faster decline in renal function, underscoring the role of total kidney volume (TKV) as an informative marker of disease severity and progression [1]. In an effort to qualify TKV as a surrogate endpoint for ADPKD clinical trials, the Polycystic Kidney Disease Outcomes Consortium engaged in a process with the FDA and the European Medicines Agency to formally qualify $\mathrm{TKV}$ as a prognostic biomarker. Using observational data from 5 different sources (University of Colorado-Denver, Mayo Clinic, Emory University, CRISP1, and CRISP2), that included a total of 2,355 patients with ADPKD with TKV determinations collected up to 30 years of follow-up, they showed that TKV was the most important prognostic term for 30\% decline in estimated GFR (eGFR) in ADPKD patients with and without preserved baseline eGFR. As a result, TKV was formally qualified, both by FDA and European Medicines Agency, as a prognostic enrichment biomarker for selecting patients at high risk for a progressive decline in renal function for inclusion in interventional clinical trials [2].

The qualification of TKV as a prognostic enrichment biomarker for ADPKD clinical trials sparks the need for establishing the best way for its estimation. In this issue 
of the American Journal of Nephrology, Magistroni and colleagues reviewed the different imaging modalities and processing techniques for estimating kidney and cyst volume, and models that include TKV measurements for estimating disease severity and progression [3]. This well written review discusses several imaging modalities and post-processing methodologies to determine TKV, with different levels of precision.

Ultrasound (US) is the commonest and most readily available imaging modality for establishing an ADPKD diagnosis. However, its use for determining disease progression is limited due to being high operator dependent and the use of the ellipsoid equation to estimate TKV, resulting in a less reproducible TKV estimation with higher coefficient of variation. Nevertheless, the wide availability and low cost associated with US makes it a good alternative for establishing disease severity in cases where magnetic resonance imaging (MRI) is not an option. Computed tomography (CT) and MRI yield TKV estimations with similar accuracy and inter/intra observer variability, being MRI the preferred imaging modality to follow-up ADPKD patients as it does not expose them to radiation. Furthermore, MRI provides excellent soft tissue contrast and anatomic detail without IV contrast, which allows for easier cystic volume estimations. Quick TKV estimations can be easily achieved from CT and MR images using the ellipsoid equation when the there is less need for accuracy such as to estimate disease severity. On the other hand, if the goal is to determine a therapeutic effect where high accuracy is required, stereology or segmentation techniques such as manual planimetry, semiautomatic or automatic techniques are needed.

Despite the role of TKV as prognostic biomarker, there is controversy on its applicability to all patients with ADPKD. Indeed, it has been shown that in some atypical cases, TKV is not the best predictor of disease progression. In an attempt to improve the prognostic value of $\mathrm{TKV}$, an imaging classification of ADPKD has been developed and validated [4]. Briefly, this classification con- sists of the application of CT and MR images to assign patients as class 1 (typical, bilateral diffuse presentation, $\sim 95 \%$ of the ADPKD population) or class 2 (atypical, asymmetric cyst distribution, $~ 5 \%$ of the ADPKD population) based on pre-specified imaging findings. In class 1 , a longitudinal mixed effect regression model to predict future eGFR decline showed that height adjusted TKV and age were the only parameters to significantly interact with time. Further stratification into A, B, C, D and E, based on height adjusted TKV and age, showed that the rate of eGFR decline and renal survival were significantly different among them, with patients in class $\mathrm{C}, \mathrm{D}$ and $\mathrm{E}$ at highest risk for eGFR decline. Contrarily, htTKV and age were not significant predictors of change in eGFR over time in class 2 patients. More recently, results from the CRISP III study with a median of 13 years of followup, showed that htTKV was an independent predictor of $a \geq 30$ and $\geq 57 \%$ decline in eGFR from baseline, with a significant correlation between the annual rate of htTKV increase and eGFR decline. Interestingly, the study showed that while the genotype was associated with CKD outcomes, it was not an independent prognostic factor after adjusting for htTKV [5].

Taken together, clinical trials indicate that TKV is an important prognostic biomarker in most patients with ADPKD. Currently, there are several imaging modalities and post-processing methodologies to determine TKV. The decision on which one to select should be based on several aspects such as purpose of the measurements, availability, and expertise.

\section{Disclosure Statement}

The authors declare that there are no conflicts of interest.

\section{Funding Source}

The authors declare that there are no funding.

\section{References}

1 Grantham JJ, et al: Volume progression in polycystic kidney disease. N Engl J Med 2006; 354:2122-2130

2 Perrone RD, et al: Total kidney volume is a prognostic biomarker of renal function decline and progression to end-stage renal disease in patients with Autosomal Dominant Polycystic Kidney Disease. Kidney Int Rep 2017;2:442-450.
3 Magistroni R, et al: A review of the imaging techniques for measuring kidney and cyst volume in establishing Autosomal Dominant Polycystic Kidney Disease (ADPKD) progression. Am J Nephrol 2018;48:67-78.
4 Irazabal MV, et al: Imaging classification of autosomal dominant polycystic kidney disease: a simple model for selecting patients for clinical trials. J Am Soc Nephrol 2015;26:160-172.

$5 \mathrm{Yu}$ ASL, et al: Baseline total kidney volume and the rate of kidney growth are associated with chronic kidney disease progression in Autosomal Dominant Polycystic Kidney Disease. Kidney Int 2018;93:691-699. 